\title{
Letalidad y la mortalidad de Covid 19 en 60 países afectados y su impacto en los aspectos demográficos, económicos y de salud
}

\author{
Covid 19 fatality and mortality in 60 affected countries and their impact on demographic, economic and \\ health aspects
}

\author{
Javier Cieza Zevallos ${ }^{1, a}$, Celene Uriol Lescano ${ }^{1, b}$
}

RESUMEN

Hay estudios que permiten comprender aspectos relacionados a la Covid 19, pero no hay muchas publicaciones relativas a su impacto poblacional. Objetivo: Estudiar la relación de la letalidad y mortalidad con variables sociales, demográficas, económicas y de salud más relevantes durante los primeros 90 días de la pandemia en 60 países del mundo de los cuatro continentes. Material y métodos: Estudio transversal descriptivo y analítico de casos. Los casos lo conformaron 60 países seleccionados según la magnitud de su afectación por Covid 19. La mortalidad y la letalidad fueron estratificadas y se contrastaron con las variables de los países seleccionados mediante comparación de medias. Resultados: Se encontró una fuerte correlación entre la mortalidad y letalidad $(\mathrm{r}=0,70)$. El día en que se inició la enfermedad en un país y su crecimiento de infectados mostraron diferencias entre países: aquellos con mejores indicadores económicos e índice de desarrollo humano, tuvieron menos letalidad al inicio de la enfermedad. Al aumentar la incidencia, estas diferencias desaparecieron. Conclusión: Los resultados sugieren que al inicio de la enfermedad en un país, los mejores servicios son importantes, pero luego cuando la enfermedad ya se expandió, la incidencia es la variable más importante. Este resultado mostró que no hay recursos sanitarios que puedan disminuir la letalidad, independiente de cualquier otra característica del país afectado.

PALABRAS CLAVE: Infecciones por coronavirus, mortalidad, economía, sistemas de salud. (Fuente: DeCS BIREME).

\section{SUMMARY}

There are studies that allow us to understand specific aspects related to COVID-19, but few studies have evaluated the population implications of it. Objectives: To study the relationship between case fatality rate and mortality with sociodemographic, economic and health variables during the first 90 day of the pandemic in 60 countries across four continents. Methods: A cross-sectional study was carried-out; cases were made up of 60 countries selected according to the magnitude of their affectation by Covid 19. Mortality and lethality were stratified and contrasted with the variables of the selected countries by means of comparison of means. Results: A strong correlation was found between mortality and lethality $(\mathrm{r}=0.70)$. The day the disease began in a country and its growth in infected showed differences between countries: those with better economic indicators and human development index had less fatality at the beginning of the disease. As the incidence increased, these differences disappeared. Conclusion:

\footnotetext{
Facultad de Medicina Alberto Hurtado, Universidad Peruana Cayetano Heredia. Perú.

Médico Especialista en Nefrología.

Médico Cirujano.
} 
The results suggest that at the beginning of the disease in a country, the best services are important, but later when the disease has already spread, incidence is the most important variable. This result showed that there are no health resources that can reduce fatality, independent of any other characteristic of the affected

KEYWORDS: Coronavirus infections, mortality, economics, health systems. (Source: MeSH NLM).

\section{INTRODUCCIÓN}

Muchos de los estudios publicados hastala redacción final de este trabajo en relación a la pandemia mundial generada por el virus SARS-CoV-2 que la Organización Mundial de la Salud (OMS) definió oficialmente como Covid 19, están en áreas relacionadas a la biología, inmunología, manifestaciones clínicas y pruebas terapéuticas, viendo al individuo como el centro protagónico de la enfermedad, situación correcta. Sin embargo, en un segundo plano ha quedado el impacto en las poblaciones de los países, cuyas diversas particularidades llevan a veces a generar supuestos en relación al alcance logrado por la enfermedad (1).

Los estudios publicados ayudan al entendimiento, manejo y toma de decisiones en los pacientes. Pero variables relacionadas a los aspectos demográficos, económicos, políticas de salud, enfermedades frecuentes en las poblaciones con las características de la mortalidad y letalidad, podrían servir para reflexionar sobre un impacto a gran escala en un horizonte futuro inmediato, mediato y de largo plazo.

En retrospectiva para contextualizar el aporte de Sir William Farr que mencionaba: "La tasa de mortalidad es un hecho; cualquier cosa más allá de esto es una inferencia”. En la actual pandemia, tenemos que utilizar los datos sobre muertes para predecir el pico de enfermedad y poder así, reforzar el aislamiento social indispensable para contenerla y optimizar los recursos del Sistema de Salud en cada país (2). Al presente debemos aceptar que puntos de vista a posteriori pueden tener un retraso de tiempo desde la introducción de la infección en un país hasta su impacto social entre 21 a 28 días.

Al utilizar las tasas de mortalidad y letalidad podríamos comparar las variables sociales, demográfica, económicas de salud más relevantes relacionadas a estas tasas y así apreciar cuáles influyeron en su mayor crecimiento y que puedan permitirnos mirar este gran problema de la humanidad desde otro punto de vista que pueda ser útil para esta pandemia u otras que podrían presentarse a futuro.

El objetivo del trabajo fue estudiar diversas variables propias de varios países del mundo relacionadas con la letalidad y mortalidad por la pandemia del Covid 19 en los primeros 60 días contados a partir del 22 de enero del 2020, fecha en que diversas bases de datos fueron acopiando información sobre casos y muertos en cada país.

\section{MATERIAL Y MÉTODOS}

Estudio transversal descriptivo y analítico de casos. Los casos lo conformaron 60 países seleccionados según la magnitud de su afectación por Covid 19, considerándose aquellos que tuvieran al menos 100 casos informados a la fecha de cierre del presente estudio (21 de abril del 2020). En todos se calculó la letalidad al tener 100, 500, $1000,2000,3000,5$ $000,10000,15000$ y 100000 casos confirmados y al cierre del estudio y su mortalidad (muertos/millón de habitantes).

Los datos fueron recopilados de fuentes formales de Salud del mundo como la OMS y la Organización Panamericana de la Salud (OPS), y en algunos países las emisiones formales del Ministerio de Salud local. (3).

Las variables estudiadas de cada país se obtuvieron del Banco Mundial, de la OMS / OPS, base de datos de KNOEMA e INDEX MUNDI. Estas variables fueron de orden económicas (PBI per cápita), turísticas (gastos en turismo exterior y arribos turísticos al país), demográficas (población, densidad poblacional, esperanza de vida al nacer, porcentaje de mayores de 65 y 75 años), sanitarias (gasto en salud per cápita, camas por mil habitantes, prevalencia de VIH, mortalidad por TBC porcentaje de adultos diabéticos y obesos), de costumbres y hábitos de la población adulta (consumo de alcohol, tabaco e inactividad física) de mortalidad (mortalidad por enfermedades cardiovasculares, por enfermedades respiratorias crónica y por cáncer; también mortalidad por enfermedades transmisibles, 
no transmisibles y por lesiones). También se analizó en función de indicadores compuestos (indicador de desarrollo humano e indicador de felicidad) Estas variables fueron contrastadas contra la magnitud de casos y magnitud de muertos como valor absoluto, mortalidad y letalidad por Covid 19 para saber si existe alguna o algunas relaciones relevantes $(4,5)$.

La mortalidad y la letalidad fueron estratificadas y se contrastaron con las variables de los países seleccionados mediante comparación de medias usando el método estadístico ANOVA y su significancia estadística $(p<0,05)$. También se consideró la eta cuadrado como prueba estadística para complementar el test de ANOVA.

El estudio no fue revisado por un Comité de ética, por tratarse de una evaluación de datos secundarios.

\section{RESULTADOS}

Los países estudiados y la magnitud de casos confirmados a la fecha de cierre del estudio se presentan en la tabla 1, se encontraron diferencias estadísticamente significativas tanto para la cantidad de casos ( $\mathrm{p}=0,001$; eta cuadrado 0,299$)$ como para la de muertos $(\mathrm{p}=0,012$; eta cuadrado $=0,231)$ entre la media de las regiones.

La correlación entre casos, muertos, letalidad, incidencia y mortalidad se presentan en la tabla 2 . La letalidad a la fecha de cierre del estudio no tuvo relación con los casos, pero tuvo una buena correlación con la mortalidad $(\mathrm{r}=0,70)$ y limitada con los muertos $(\mathrm{r}=0,48)$. Los mejores índices de correlación lo tuvieron la magnitud de casos y muertos registrados $(\mathrm{r}=0,90)$ y la incidencia y la mortalidad.

En la tabla 3 se muestran las variables que tuvieron diferencia en sus promedios respecto a la letalidad estratificada ( $>4 \%$, entre 2 y $4 \%$, entre 0,01 y $2 \%$ y $0 \%$ ). Al analizar los países que tuvieron 10000 casos al cierre del estudio en función de la letalidad estratificada en un corte al $4 \%$, se encontró como variable diferenciable el índice de desarrollo humano.

Al cierre del estudio la letalidad estratificada (mayor y menor del 4\%), considerando solamente los países con más de 10000 casos, solo mostraron significancia estadística con la incidencia y el día que transcurrió desde el 22 de enero. Los países con letalidad mayor de $4 \%$ tuvieron una incidencia de 1 $942 \pm 1309$ casos/millón de habitantes y los países con letalidad menor de $4 \%$ tuvieron una incidencia de $859 \pm 706$ casos/millón de habitantes $(\mathrm{p}=0,015)$. En los países que tuvieron letalidad de más de $4 \%$ su primer fallecido fue registrado el día $38 \pm 15$, mientras que en los que la letalidad fue menor de $4 \%$, su primer muerto se registró en el día $49 \pm 11(\mathrm{p}=0,043)$.

El gráfico 1 muestra los cambios de la letalidad

Tabla 1. Países incluidos en el estudio según regiones y magnitud de casos y muertos.

\begin{tabular}{|c|c|c|c|}
\hline Región & Países & $\begin{array}{l}\text { Promedio } \\
\text { Casos }\end{array}$ & $\begin{array}{c}\text { Promedio } \\
\text { Muertos }\end{array}$ \\
\hline Europa (21) & $\begin{array}{l}\text { Alemania, Austria, Bélgica, Dinamarca, España, } \\
\text { Finlandia, Francia, Grecia, Italia, Noruega, Países } \\
\text { Bajos, Portugal, Reino Unido, República Checa, } \\
\text { Rumanía, Rusia, Serbia, Suecia, Suiza, Turquía, } \\
\text { Ucrania. }\end{array}$ & $52733 \pm 63336$ & $4974 \pm 7938$ \\
\hline América del Norte (3) & Estados Unidos, Canadá, México. & $2745597 \pm 436354$ & $14656 \pm 23318$ \\
\hline América Central (7) & $\begin{array}{l}\text { Costa Rica, Cuba, El Salvador, Guatemala, } \\
\text { Honduras, Panamá, República Dominicana. }\end{array}$ & $1737 \pm 2058$ & $66 \pm 85$ \\
\hline América Sur (9) & $\begin{array}{l}\text { Argentina, Bolivia, Brasil, Colombia, Ecuador, } \\
\text { Chile, Paraguay, Perú, Uruguay }\end{array}$ & $9508 \pm 12924$ & $441 \pm 797$ \\
\hline Asia (13) & $\begin{array}{l}\text { Arabia Saudita, China, Corea del Sur, Filipinas, } \\
\text { India, Indonesia, Irán, Israel, Japón, Malasia, } \\
\text { Pakistán, Singapur, Tailandia }\end{array}$ & $20627 \pm 27994$ & $961 \pm 1770$ \\
\hline África y Oceanía (7) & $\begin{array}{l}\text { Argelia, Australia, Burkina Faso, Camerún, Egipto, } \\
\text { Nueva Zelanda, Sudáfrica }\end{array}$ & $2705 \pm 2019$ & $121 \pm 140$ \\
\hline
\end{tabular}


Tabla 2. Correlación entre los casos registrados, muertos, incidencia, mortalidad y letalidad al 21 de abril del 2020.

\begin{tabular}{|c|c|c|c|c|c|c|c|c|}
\hline & \multicolumn{2}{|c|}{ Muertos } & \multicolumn{2}{|c|}{ Letalidad } & \multicolumn{2}{|c|}{ Incidencia } & \multicolumn{2}{|c|}{ Mortalidad } \\
\hline & $\mathbf{r}$ & $\mathbf{p}$ & $\mathbf{r}$ & $\mathbf{p}$ & $\mathbf{r}$ & p & $\mathbf{r}$ & $\mathbf{p}$ \\
\hline Casos & 0,90 & 0,00 & 0,21 & 0,11 & 0,46 & 0,00 & 0,37 & 0,00 \\
\hline Muertos & & & 0,48 & 0,00 & 0,60 & 0,00 & 0,64 & 0,00 \\
\hline Letalidad & & & & & 0,42 & 0,00 & 0,70 & 0,00 \\
\hline Incidencia & & & & & & & 0,86 & 0,00 \\
\hline
\end{tabular}

Tabla 3. Variables con significancia estadística asociadas a la letalidad cuando los países registraron sus primeros 100 casos.

\begin{tabular}{|c|c|c|c|c|c|c|c|c|c|c|}
\hline & \multicolumn{8}{|c|}{ Letalidad en los 100 primeros casos } & \multirow{3}{*}{$p$} & \multirow{3}{*}{$\begin{array}{c}\text { eta } \\
\text { cuadrado }\end{array}$} \\
\hline & \multicolumn{2}{|c|}{$>4 \%(n=8)$} & \multicolumn{2}{|c|}{$2-4 \%(n=8)$} & \multicolumn{2}{|c|}{$0,01-2 \%(n=21)$} & \multicolumn{2}{|c|}{$0,00 \%(n=23)$} & & \\
\hline & Media & DS & Media & DS & Media & DS & Media & DS & & \\
\hline $\begin{array}{l}\text { Índice de desarrollo } \\
\text { humano }\end{array}$ & 0,73 & 0,15 & 0,70 & 0,09 & 0,84 & 0,09 & 0,84 & 0,09 & 0,001 & 0,255 \\
\hline Primer muerto (día) & 44,5 & 19,2 & 56,4 & 5,7 & 43,2 & 14,5 & 57,6 & 5,1 & 0,048 & 0,251 \\
\hline $\begin{array}{l}\text { Mortalidad por } \\
\text { enfermedad respiratoria } \\
\text { crónica }\end{array}$ & 14,7 & 6,7 & 14,7 & 6,0 & 22,3 & 7,6 & 5,6 & 1,9 & 0,002 & 0,226 \\
\hline Mortalidad por lesiones & 10,1 & 3,7 & 9,4 & 6,1 & 9.4 & 4,9 & 5,6 & 2,0 & 0,008 & 0,190 \\
\hline Esperanza de vida (años) & 74,0 & 6,6 & 73,0 & 6,2 & 79,1 & 4,2 & 78,3 & 23 & 0,011 & 0,178 \\
\hline Índice de Felicidad & 5,7 & 0,8 & 5,4 & 0,8 & 6,2 & 0,9 & 6,5 & 0,8 & 0,018 & 0,165 \\
\hline Mayores de 65 años (\%) & 9,4 & 6,8 & 8,7 & 5,4 & 13,3 & 6,9 & 15,8 & 5,9 & 0,021 & 0,158 \\
\hline PBI per cápita & 14614 & 19525 & 6624 & 2671 & 27257 & 21206 & 30099 & 24149 & 0,017 & 0,164 \\
\hline
\end{tabular}

PBI: Producto bruto interno

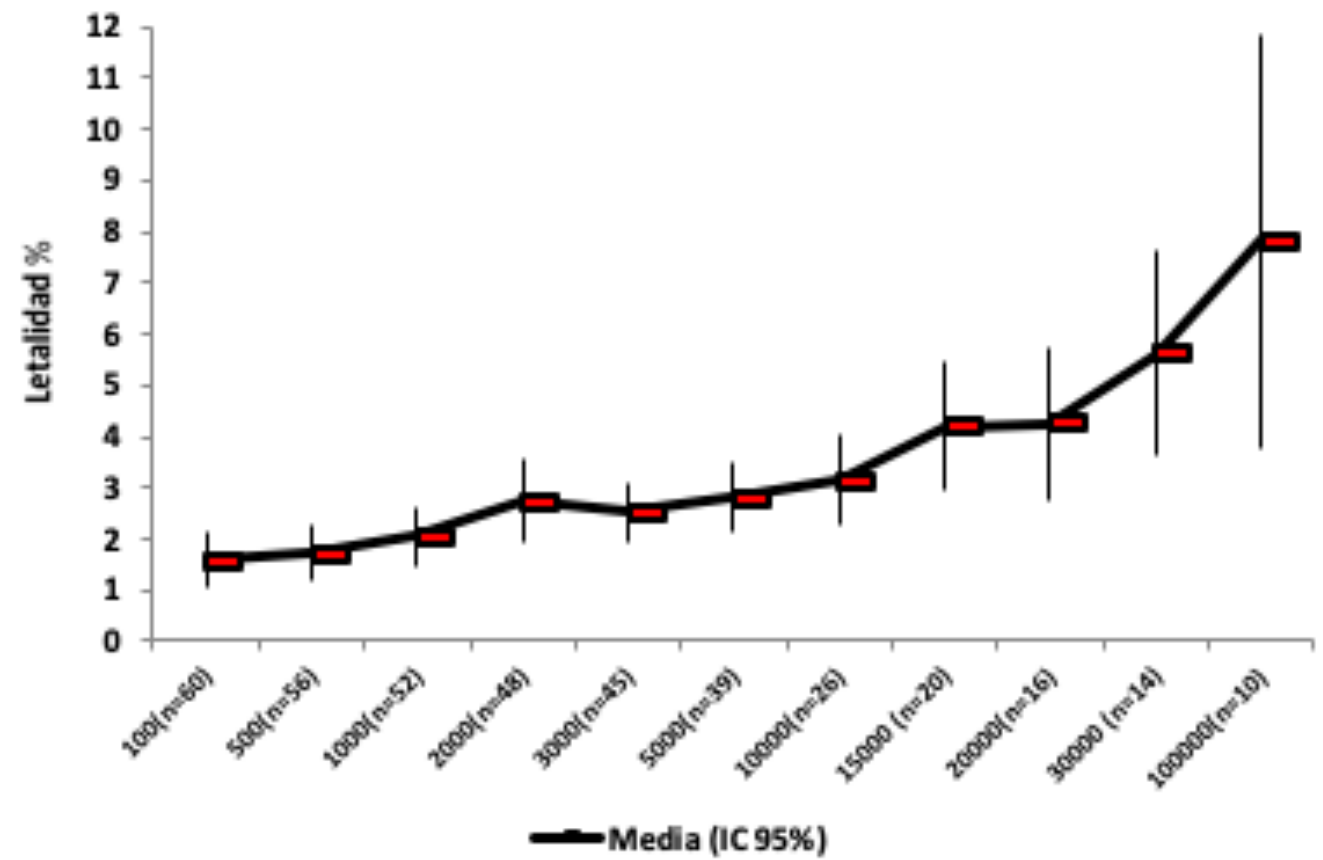

Gráfico 1. Letalidad del Covid 19 entre el 21 de enero al 21 de abril del 2020 desde que tuvieron más de cien casos confirmados hasta cuando alcanzaron los cien mil casos. 
en función de los casos registrados en los países estudiados. Se observa que según aumentan los casos confirmados hay un incremento de la letalidad.

Las variables que tuvieron diferencias significativas al analizar la mortalidad estratificada en más de 50 muertos/millón habitantes, entre 10 y 50 , entre 2 y 10 y menos de 2 muertos/millón de habitantes, se muestran en la tabla 4.

DISCUSIÓN
La letalidad y mortalidad, como ya se comentó, son datos tangibles que se han contrastado con las características de los países estudiados mencionadas en la sección material y métodos. La tasa de mortalidad permitió realizar un análisis de la situación en salud de una determinada región en un tiempo determinado como el contexto actual de la pandemia de Covid 19 (6).

Se seleccionaron 60 países por conveniencia,

Tabla 4. Variables con significancia estadística asociadas a la letalidad cuando los países registraron 20000 casos.

\begin{tabular}{|c|c|c|c|c|c|c|}
\hline & \multicolumn{6}{|c|}{ Letalidad al alcanzar los 20000 casos de COVID 19} \\
\hline & \multicolumn{2}{|c|}{$>4 \%(n=6)$} & \multicolumn{2}{|c|}{$\leq 4 \%(n=10)$} & \multirow{2}{*}{$\mathbf{p}$} & \multirow{2}{*}{$\begin{array}{c}\text { eta } \\
\text { cuadrado }\end{array}$} \\
\hline & Media & DS & Media & DS & & \\
\hline Adultos desempleados (\%) & 10,0 & 5,0 & 5,7 & 2,7 & 0,038 & 0,273 \\
\hline Camas por 1000 habitantes & 2,90 & 1,03 & 4,98 & 2,19 & 0.048 & 0.251 \\
\hline
\end{tabular}

Tabla 5. Variables relacionadas estadísticamente a la mortalidad el día 21 de abril del 2020.

\begin{tabular}{|c|c|c|c|c|c|c|}
\hline \multirow[b]{2}{*}{$\begin{array}{l}\text { Variables con significancia } \\
\text { estadística }\end{array}$} & \multicolumn{6}{|c|}{ Mortalidad (muertos/millón habitantes) } \\
\hline & $>50(n=14)$ & $\begin{array}{l}10 \text { a } 50 \\
(n=14)\end{array}$ & 2 a $10(n=20)$ & $<2(n=12)$ & $p$ & $\begin{array}{c}\text { eta } \\
\text { cuadrado }\end{array}$ \\
\hline $\begin{array}{l}\text { Mortalidad (enfermedades } \\
\text { transmisibles) (\%) }\end{array}$ & $8,29 \pm 4.94$ & $6.43 \pm 5,05$ & $10,5 \pm 5,98$ & $26,7 \pm 16,31$ & 0,00 & 0,44 \\
\hline Gasto en salud per cápita & $4631 \pm 2657$ & $2080 \pm 2050$ & $1087 \pm 1286$ & $859 \pm 1241$ & 0,00 & 0,40 \\
\hline Índice Desarrollo Humano & $0,91 \pm 0,04$ & $0,84 \pm 0,07$ & $0,80 \pm 0,09$ & $0,70 \pm 0,02$ & 0,00 & 0,38 \\
\hline Población más de 75 años (\%) & $8,86 \pm 2,33$ & $6.0 \pm 2,80$ & $4,24 \pm 2,37$ & $3,26 \pm 3,73$ & 0,00 & 0,37 \\
\hline Pobreza (ingreso< $\$ 5 /$ día) & $0,83 \pm 0,93$ & $4,04 \pm 3,95$ & $8,68 \pm 9,27$ & $21,00 \pm 18,70$ & 0,00 & 0,36 \\
\hline PBI per cápita ( $\$$ constantes) & $45155 \pm 16513$ & $28824 \pm 2449$ & $13782 \pm 13813$ & $14102 \pm 20385$ & 0,00 & 0,33 \\
\hline Alfabetos adultos (\%) & $97,99 \pm 3,68$ & $96,4 \pm 2,78$ & $94,69 \pm 7,18$ & $81,47 \pm 16,97$ & 0,00 & 0,33 \\
\hline Esperanza de vida (años) & $81,6 \pm 3,01$ & $78,7 \pm 3,08$ & $76,14 \pm 3,77$ & $72,56 \pm 8,39$ & 0,00 & 0,32 \\
\hline $\begin{array}{l}\text { Mortalidad por enfermedad } \\
\text { respiratoria crónica }(\%)\end{array}$ & $26,36 \pm 4,27$ & $22,2 \pm 4,85$ & $18,7 \pm 7,03$ & $15 \pm 8,84$ & 0,00 & 0,29 \\
\hline $\begin{array}{l}\text { Mortalidad por TBC } \\
(* 100000 \text { habitantes })\end{array}$ & $0,55 \pm 0,45$ & $2,07 \pm 2,27$ & $6,18 \pm 9,07$ & $12,84 \pm 13,79$ & 0,00 & 0,24 \\
\hline $\begin{array}{l}\text { Arribos turísticos } \\
\text { (millones/año) }\end{array}$ & $35,81 \pm 30,23$ & $\begin{array}{c}11,145 \pm \\
12,83\end{array}$ & $13,79 \pm 15,32$ & $10,15 \pm 12,94$ & 0,00 & 0,23 \\
\hline Índice de Felicidad & $6,74 \pm 0,83$ & $6,36 \pm 0,84$ & $5,85 \pm 0,80$ & $5,67 \pm 0,88$ & 0,01 & 0,21 \\
\hline Obesidad adultos (\%) & $22,86 \pm 4,56$ & $23,99 \pm 3,82$ & $22,16 \pm 9,38$ & $14,16 \pm 9,17$ & 0,01 & 0,20 \\
\hline Consumo alcohol adultos (\%) & $10,07 \pm 3.02$ & $8,14 \pm 3,48$ & $6,4 \pm 4,06$ & $5,66 \pm 3,05$ & 0,01 & 0,19 \\
\hline $\begin{array}{l}\text { Mortalidad } \\
\text { (enfermedad cardiovascular) }\end{array}$ & $31,36 \pm 6.06$ & $33,86 \pm 11,01$ & $34,1 \pm 10,32$ & $23,08 \pm 6,39$ & 0,01 & 0,19 \\
\hline
\end{tabular}


considerando los cinco continentes y la diferente magnitud de afectación según los casos y muertos informados. El criterio se pensó, en responder si había relaciones entre el impacto de la pandemia y las características propias de países que se relacionen o asocien con la letalidad y mortalidad. Esto reflejaría indirectamente la respuesta de cada país ante esta situación de esta enfermedad tan peculiar como lo es pandemia por Covid 19.

La primera observación es la fuerte correlación entre el número de casos y muertos y el de incidencia y mortalidad (tabla 2), que reflejan un mismo concepto que es el de afectación a personas traducidas a poblaciones de sus países e indirectamente indica que la afectación ha ocurrido siguiendo reglas universales independiente de las características de los países estudiados. En esta misma línea de reflexión, es sobresaliente notar que la letalidad no tiene asociación con la magnitud de casos registrados, pero sí con la mortalidad. Esto permite sostener que los procesos mortales de los afectados, carece hasta esta publicación, de mecanismo terapéutico exitoso. Puede observarse que el incremento de casos se acompaña del incremento de la letalidad (gráfico 1).

Si bien no hubo correlación lineal entre casos y letalidad, al estratificarse ésta según momentos ajustados a magnitud de casos afectados en cada país, cuando un país inicia el proceso de infectados se observaron diferencias entre países. Los países con mejores indicadores como PBI per cápita, índice de desarrollo humano, esperanza de vida, porcentaje de personas con más de 60 años, menor mortalidad por enfermedades respiratorias crónica y ocurrencia más tardía de su primera muerte prácticamente no tuvieron muertos por Covid 19. Los países con mayor mortalidad por lesiones (violencia, conflictos armados, accidentes) tuvieron más letalidad temprana. Sin embargo, al aumentar la incidencia, estas diferencias ya se pierden y por ejemplo, cuando se alcanzan 20 000 casos el mayor desempleo y la menor proporción de camas/1000 habitantes son los aspectos relevantes asociados a mayor letalidad.

Estos cambios en las variables asociadas a la letalidad según la incidencia de la enfermedad en cada país traducen que al comienza de la propagación de la enfermedad en un país, las características de sus servicios son importantes, cuando la enfermedad ya afecta muchas personas, la incidencia es la variable más importante. Este resultado sí nos parece sumamente importante pues traduce que no hay recursos sanitarios que puedan disminuir la letalidad independiente de cualquier otra característica del país afectado. Es altamente probable que esto tenga varias interpretaciones, pero la más viable hasta la fecha de este manuscrito radique en el fisiopatológico grave, probablemente por falla multiorgánica que sabemos que cuando ocurre la muerte es inminente y que en esta enfermedad ocurre rápidamente.

Al analizar los resultados como afectación mortal en las poblaciones (mortalidad), se puede apreciar que ésta se relacionó inversamente con la mortalidad por enfermedades transmisibles, con la pobreza y la mortalidad por tuberculosis (tabla 5). Las demás variables gasto en salud y PBI per cápita así como también mayor desarrollo humano, menor alfabetismo en adultos, mayor esperanza de vida, mayor porcentaje de personas con más de 75 años, mayor porcentaje de muertes por enfermedades respiratorias crónicas y cardiovasculares entre otras, como también mayor consumo de alcohol, tuvieron relación directa. Debemos resaltar que la mayor esperanza de vida se acompaña de mayor porcentaje de personas con enfermedades respiratorias crónicas y cardiovasculares lo que conlleva a mayor gasto en salud per cápita. También es muy probable que el mayor PBI per cápita se asocie a mayor consumo de alcohol, mayor obesidad y más movimiento turístico $(7,8)$.

De esta manera la letalidad según el crecimiento de la incidencia o casos en un país, parece ser menor en los países con mejor capacidad económica en general al comienzo de la propagación de la enfermedad para luego solamente corresponder a la magnitud de la incidencia. Pero finalmente la mayor mortalidad parece corresponder a ciertas características de los países con más capacidad económica, como costumbres y envejecimiento.

Estudios realizados por la OMS, en los diferentes continentes, en relación a los sistemas de salud implementados, ya advertían de sus deficiencias y, al parecer, la pandemia por Covid 19 se ha encargado de ventilar la pobre respuesta de estos, las carencias y fallas, independientemente del país donde haya acontecido.

Las reformas en los sistemas de salud son promovidas, mejoradas con mayor énfasis, luego de la Declaración de Alma- Ata, que considera la salud 
como derecho humano básico. Pero el concepto de salud enfocado únicamente desde un punto de vista materialista monetario podría ser un enfoque errado. En países como los de Sudamérica, las reformas en salud se han venido implementando desde los años noventa, la mayoría enfocados a reforzar el primer nivel de atención, lograr la igualdad en salud y brindar un financiamiento del Estado acorde a sus necesidades.

Por otro lado, sistemas de salud como el de Estados Unidos de América, no brindan un seguro subsidiado por el estado, existe una inequidad notoria para poder acceder a un servicio de salud. En Europa, existe un mayor gasto en salud que en otros continentes, esto relacionado a su mayor PBI. En África y Oceanía, no se logra cubrir la demanda en salud; según la OMS, África posee el $25 \%$ de la carga de morbilidad mundial, pero su parte del gasto mundial en salud es inferior al $1 \%$. Finalmente, en Asia, a pesar de ser el destino de inversión sanitaria con más rápido crecimiento en el mundo, existen expectativas cambiantes de los consumidores que conducirían inevitablemente a cambios demográficos e implicarían una inequidad en salud para la población $(9,10,11,12)$.

Por lo expuesto, se genera una situación bastante debatible, que es el presuponer que si un país posee un mayor PBI, realiza un mayor gasto en salud y sus habitantes tienen un elevado índice de desarrollo humano, la tasa de mortalidad sería menor. Siendo todo lo contrario, podemos inferir varias situaciones, algunas de las cuales se relacionan con la pirámide poblacional de cada país. En el caso de Europa, al tener una población más longeva, realiza un mayor gasto en salud, dada la estrecha relación con el aumento de morbilidades, el consumo de fármacos y el alargamiento de la vida de las personas ancianas. Por otra parte, existe también una desigualdad enorme en relación al acceso a los servicios de salud, ya que muchos de los sistemas de salud ya se encontraban sobrecargados antes de la epidemia, con pacientes con comorbilidades, importantes, y cuya salud se ha visto comprometida de sobremanera en esta situación.

En conclusión, este trabajo abre temas que debemos profundizar bajo un horizonte de mejora de la vida del ciudadano antes que el puramente monetario, lo que implica cambios en los conceptos de educación de los ciudadanos que involucra la salud en una visión integral y no únicamente como ausencia de enfermedad doliente sentida que impide la vida placentera puramente material.

Declaración de financiamiento y de conflictos de interés:

El estudio fue financiado por los autores quienes niegan cualquier tipo de conflicto de interés.

\section{Contribución de autoría:}

JCZ: Elaboración de trabajo, diseño, recolección de datos, análisis y escritura de manuscrito. CUL: Elaboración de idea, diseño, recolección de datos, análisis y escritura de manuscrito.

\section{Correspondencia:}

Celene Taís Uriol Lescano

Dirección: Calle Santa Honorata 589. Lima, Perú.

Correo electrónico: celene.uriol@upch.pe

Celular: 958476087

\section{REFERENCIAS BIBLIOGRÁFICAS}

1. World Health Organization. Nuevo Coronavirus 2019. Ginebra: World Health Organization; 2020. Disponible en: https://www.who.int/es/emergencies/ diseases/novel-coronavirus-2019? gclid=EAIaIQob ChMIprjHjI746QIVWtyGCh0AUQSzEAAYASAA EgLOY_D_BwE 10/06/2020

2. Centre for Evidence-Based Medicine. 2020. COVID 19: William Farr's way out of the pandemic. Inglaterra: Centre for Evidence-Based Medicine; 2020 (Citado el 10 de junio de 2020) Disponible en: https://www.cebm.net/Covid 19/Covid 19-williamfarrs-way-out-of-the-pandemic/

3. Organización Mundial de la Salud. Publicaciones y fuentes de información. Ginebra: Organización Mundial de la Salud; 2020. (Citado el 10 de junio de 2020) Disponible en: https://www.who.int/pehemf/publications/es/

4. Knoema. Datos gratuitos, estadísticas, análisis, visualizacion \& para compartir. New York: Knoema; 2020. (Citado el 10 de junio de 2020) Disponible en: https://knoema.es/

5. IndexMundi. Indicadores mundiales. North Carolina, USA: Indexmundi; 2020 (Citado el 10 de junio de 2020) Disponible en: https://www.indexmundi.com/ es/datos/

6. Organización Panamericana de la Salud. Enfermedad por el Coronavirus ('COVID 19)". Washington DC: Organización Panamericana de la Salud; 2020. (Citado el 10 de junio de 2020) Disponible en: https:// www.paho.org/es/temas/coronavirus/enfermedadpor-coronavirus-Covid-19?gclid=EAIaIQobChMI5 9WQyKX46QIVB2KGCh0NwgDcEAAYASAAEgI jF_D_BwE

7. Instituto Vasco de Estadística. Índice de Desarrollo 
Humano por indicadores según países. Araba, Esapaña: Instituto Vasco de Estadística; 2019. (Citado el 10 de junio de 2020) Disponible en: https://www.eustat.eus/elementos/ele0013500/ ti_Indice_de_Desarrollo_Humano_por_indicadores segun_paises_2019/tbl0013566_c.html

8. King's Critical Care. Evidence Summary Clinical Management of COVID 19; 2020 (Citado el 10 de junio de 2020) Disponible en https://www.nwpgmd. nhs.uk/sites/default/files/KCC\%20COVID19\%20 Evidence\%20Summary.pdf

9. Báscolo E, Houghton N, Del Riego A. Types of health systems reforms in Latin America and results in health access andcoverage. Rev Panam Salud Publica. 2018; 42:1-9.

10. Grigorov V. Healthcare in Africa. Cardiovasc J Afr.
2009; 20(5): 275-277.

11. World Health Organization. Health systems Ginebra: World Health Organization; 2020. (Citado el 10 de junio de 2020) Disponible en: http://www.euro.who. int/en/health-topics/Health-systems

12. Bain \& Company.Asia-PacificFrontLine ofHealthcare Report 2020. Bain \& Company; 2020. (Citado el 10 dejuniode2020)Disponibleen:https:/www.bain.com/ contentassets/a1d1395b809d424a8db679657f95 b19d/bain_report_asia-pacific_front_line_of healthcare.pdf

Recibido: 05/07/2020 Aceptado: 27/09/2020 